\title{
Sharing Public Health Information with Non-Public Health Partners
}

\author{
Wayne Loschen*, Rekha Holtry, Kalman Hazins and Sheryl Happel Lewis \\ Johns Hopkins University Applied Physics Laboratory, Laurel, MD, USA
}

\section{Objective}

The objective of this project is to provide a technical mechanism for information to be easily and securely shared between public health ESSENCE users and non-public health partners; specifically, emergency management, law enforcement, and the first responder community. This capability allows public health officials to analyze incoming data and create interpreted information to be shared with others. These interpretations are stored securely and can be viewed by approved users and captured by authorized software systems. This project provides tools that can enhance emergency management situational awareness of public health events. It also allows external partners a mechanism for providing feedback to support public health investigations.

\section{Introduction}

Automated Electronic Disease Surveillance has become a common tool for most public health practitioners. Users of these systems can analyze and visualize data coming from hospitals, schools, and a variety of sources to determine the health of their communities. The insights that users gain from these systems would be valuable information for emergency managers, law enforcement, and other nonpublic health officials. Disseminating this information, however, can be difficult due to lack of secure tools and guidance policies. This abstract describes the development of tools necessary to support information sharing between public health and partner organizations.

\section{Methods}

The project initially brought together public health and emergency management officials to determine current gaps in technology and policy that prevent sharing of information on a consistent basis. Officials from across the National Capital Region (NCR) in Maryland, Virginia, and the District of Columbia determined that a web portal in which public health information could be securely posted on and captured by non-public health users (humans and computer systems) would be best. The development team then found open source tools, such as the Pebble blogging system, that would allow information to be posted, tagged, and searched in an easily navigable site. The sys- tem also provided RSS feeds both on the site as whole and specific tags to support notification. The team made modifications to the system to incorporate spring security features to allow the site to be securely hosted requiring usernames and passwords for access. Once the Pebble system was completed and deployed, the NCR's aggregated ESSENCE system was adapted to allow users to submit daily reports and post time series images to the new site. An additional feature was created to post visualizations every evening to the site summarizing the day's reports.

\section{Results}

The system has been in testing since March of 2012 and users of the system have provided valuable feedback. Based on the success of the tests, public health users in the NCR have begun working on the policy component of the project to determine when and how it should be used. Modifications to the system since deployment have included a single sign on capability for ESSENCE users and the desire to allow other features of ESSENCE to be posted beyond time series graphs, such as GIS maps and statistical reports.

\section{Conclusions}

Having tools that can promote exchange of information between public health and non-public health partners such as emergency management, law enforcement, and first responders can greatly enhance the situational awareness and impact overall preparedness and response. By having tools embedded in ESSENCE, users are able to integrate the information sharing aspects into their daily routines with a small amount of effort. With the use of open source tools, the same type of capability can be easily replicated in other jurisdictions. This presentation will describe the lessons learned and potential improvements the project will incorporate in the future.

\section{Keywords}

Open Source; Emergency Management; Information Sharing

*Wayne Loschen

E-mail: wayne.loschen@jhuapl.edu 